Cahiers $d u$ MONDE RUSSE

\section{Cahiers du monde russe}

Russie - Empire russe - Union soviétique et États indépendants

$50 / 4 \mid 2009$

Varia

\title{
Melanie Ilic, Jeremy Smith, eds., Soviet State and Society Under Nikita Khrushchev
}

\section{Marc Elie}

\section{(2) OpenEdition \\ Journals}

Édition électronique

URL : https://journals.openedition.org/monderusse/7193

DOI : 10.4000/monderusse. 7193

ISSN : $1777-5388$

Éditeur

Éditions de l'EHESS

Édition imprimée

Date de publication : 15 décembre 2009

Pagination : 851-854

ISBN : 978-2-7132-2261-0

ISSN : $1252-6576$

Référence électronique

Marc Elie, "Melanie llic, Jeremy Smith, eds., Soviet State and Society Under Nikita Khrushchev », Cahiers du monde russe [En ligne], 50/4 | 2009, mis en ligne le 12 janvier 2011, consulté le 02 septembre 2022. URL : http://journals.openedition.org/monderusse/7193 ; DOI : https://doi.org/ 10.4000/monderusse.7193

Ce document a été généré automatiquement le 2 septembre 2022.

Tous droits réservés 


\title{
Melanie Ilic, Jeremy Smith, eds., Soviet State and Society Under Nikita Khrushchev
}

\author{
Marc Elie
}

\section{RÉFÉRENCE}

Melanie ILIC, Jeremy SMIтH, eds., Soviet State and Society Under Nikita Khrushchev.

Londres - New York : Routledge, 2009, 216 p.

1 La période dite du dégel en Union soviétique (1953-1964) continue de susciter l'intérêt des chercheurs : de plus en plus, elle apparaît comme le pivot du « siècle soviétique ", à la fois deuxième souffle et premiers signes d'essoufflement du système. Après l'ouvrage

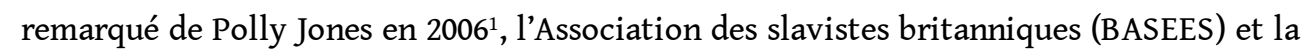
maison Routledge publient un nouveau recueil d'articles intégralement consacré aux réformes khrouchtchéviennes. Tous les contributeurs sont de jeunes chercheurs - thésards, postdoctorants ou récemment recrutés. Il est d'autant plus triste de constater que ces papiers, à l'exception des contributions de Laurent Coumel, Helen Carlbäck et Alexander Titov, n'ont pas le niveau requis pour des travaux scientifiques. Issus d'un colloque tenu fin 2007, la grande majorité des articles reposent sur un argumentaire faible et une documentation lacunaire. Ils ont été insuffisamment retravaillés pour la publication.

2 Le solide article de Laurent Coumel sur l'infortunée réforme de l'éducation de 1958 est une heureuse exception dans ce recueil. L'auteur montre comment le projet de «rapprochement de l'école et de la vie», initié par Hruščev, a été victime de la «pluralité» introduite par le dégel. Pour le Premier secrétaire, la modernisation du système scolaire hérité des années 1920-1930 passait par l'instauration d'un stage d'un an dans la production, obligatoire pour les élèves désireux d'accéder à l'enseignement supérieur. Mais des noms illustres de la science soviétique, occupant une position 
influente dans les débats officiels sur le projet de loi et dans l'application de la réforme, ont su non seulement avancer une vision concurrente de la modernité éducative, mais encore inventer des stratégies de contournement des nouvelles règles d'admission dans le supérieur. Ainsi Mihail Lavrent'ev crée Akademgorodok en se ménageant le droit de recruter lui-même les meilleurs éléments, Andrej Kolmogorov organise des internats élitistes, et d'autres universitaires mettent en place des procédures d'admission sur mesure dans leur établissement. Cette coexistence d'un ouvriérisme affiché et d'un élitisme tout aussi officiel - Hruščev lui-même soutenant l'idée d'écoles pour surdoués - manifeste l'ambiguïté des réformes du dégel.

3 Le papier d'Helen Carlbäck sur la loi de 1944 abolissant le droit des femmes à faire reconnaître leurs enfants nés hors mariage par leur père touche un aspect particulièrement important de l'histoire sociale de l'URSS des années 1940-1970. La libération de la parole concernant les dispositions de cette loi pendant le dégel a permis une controverse sociale sur un texte législatif qui, au final, « exprime l'intention plutôt conservatrice et conventionnelle de contrôler la sexualité des femmes et d'empêcher les modes de vie licencieux». Les opinions des Soviétiques sur la question de la reconnaissance de paternité et ses conséquences - les mères ne pouvaient revendiquer une aide financière du père d'enfants «illégitimes » et les enfants nés hors mariage ne pouvaient connaitre leur origine paternelle - se sont révélées très divergentes. Carlbäck montre qu'une partie de la population a soutenu l'esprit et les dispositions de la loi, avec d'intéressantes dissonances selon les républiques.

Dans un article de synthèse, Alexander Titov met en perspective le nouveau programme du parti (1961) et sa réception dans l'histoire du second siècle soviétique : « Le fossé entre les promesses du programme" et une situation économique devenue difficile « discréditèrent le projet soviétique » (p. 21), au point que « le régime eut à ranimer ses soutiens par de nouveaux moyens, par exemple en s'ouvrant sur le nationalisme russe ou bien en cherchant sa base sociale dans le culte officiel des vétérans de guerre et auprès des retraités» (p. 22). Malheureusement, le «code moral des bâtisseurs du communisme» - étonnante table de douze préceptes de bonne conduite d'inspiration communiste et humaniste - n'est pas évoqué, alors qu'il avait été placé par la direction politique au cœur du programme.

5 Pour Mark Smith, le programme de construction d'immeubles d'habitation lancé par HruŠčev en 1957 marque la naissance d'un véritable droit au logement en URSS. Alors que « l'économie du logement urbain était structurée par un discours non de droit mais de gratitude et de don » sous Stalin, « les droits des citoyens [...] au logement - c'est-àdire l'éventualité réaliste d'obtenir avec certitude un appartement familial - étaient de plus en plus largement reconnus » au tournant des années 1960 (p. 38). Pour expliquer cette transformation, Smith souligne la rivalité entre deux concepts marquants du dégel, celui de la rationalisation économique - construire le plus possible, le mieux possible et au meilleur prix - et celui du mythe de l'avènement du communisme, dont le mikrorajon est l'incarnation urbanistique. Cependant, le rapprochement que l'auteur esquisse entre l'innovation juridique du droit au logement et ce couplage entre technocratie et idéologie reste artificiel : la raison pour laquelle un surcroît d'efficacité dans la construction et la vision d'un avenir collectiviste radieux auraient pu renforcer la réalité de ce droit n'apparaît pas clairement.

6 En décortiquant les brochures officielles, Melanie Ilic décrit les fonctions que devaient remplir les «comités de femmes» (žensovety) réanimés sous Hruščev. Elle relève la 
dualité fonctionnelle de ces comités, à la fois "plateforme d'extension de la participation féminine dans l'économie nationale » et "incursion supplémentaire de l'État soviétique dans la vie privée, domestique et familiale» qui a pu apporter «des améliorations réelles et durables à la vie de nombreuses femmes au niveau local » (p. 118). Reste à vérifier à l'aide d'archives et d'entretiens la validité de cette hypothèse.

7 Julie Elkner estime que le KGB, dans les années 1950-1960, a travaillé à modifier son image afin de restaurer la confiance de la population dans les « organes ». S'appuyant en particulier sur les réunions de studios de cinéma où étaient discutés, en la présence de consultants du KGB, les scénarios des films mettant en scène les services secrets, Elkner montre comment s'est opéré le passage de la répression à la profilaktika [prévention], de l'interrogatoire musclé à la beseda [entretien], de l'indic au doverennoe lico [personne de confiance], etc. Si l'effort mis en œuvre par les services pour se créer une nouvelle image est indéniable, Elkner en exagère l'importance pour les leaders du dégel. En fait, la réforme des services secrets avait bien d'autres motivations que de s'attirer les faveurs de la population - souci de contrôler les services (et non d'être livré à eux comme sous Stalin puis Berija), de les rendre plus efficaces (réduction d'effectifs, des réseaux d'informateurs inutiles, techniques nouvelles) et de sortir du paradigme de la répression totale en agissant dans un relatif cadre légal. Des réflexions incongrues émaillent le propos d'Elkner. Ainsi, on apprend que les tchékistes auraient été marqués d'un « fort stigmate» (p. 142) dans la société soviétique poststalinienne ; que les autorités auraient même été " effrayées " par les victimes de Stalin revenant des camps, qui auraient pu leur demander des comptes (p.151); et que malgré le relookage, les tchékistes des années 1960-1970 auraient souffert d'un complexe d'infériorité à l'égard de l'intelligentsia dissidente (p. 156).

8 Pia Koivunen place le Festival mondial de la jeunesse à Moscou en 1957 dans le contexte d'une "culture de guerre froide ", où la concurrence des systèmes s'installe dans les esprits. Mais l'analyse s'enferre vite dans une quête stérile du degré d'« ouverture » que l'URSS manifeste avec le festival. La conclusion est forcément d'une portée limitée : malgré des progrès, l'URSS n'était que " partiellement ouverte » sous Hruščev (p. 61). Loin de vouloir manifester une ouverture maximale, l'objectif des autorités n'était-il pas plutôt de convaincre que le projet soviétique était bien vivant malgré les révélations du $\mathrm{XX}^{\mathrm{e}}$ Congrès et l'écrasement de la révolte hongroise l'année précédente, en présentant au monde un modèle politique, culturel, social au sommet de sa forme, dans la représentation qu'elles s'en faisaient?

Robert Hornsby tente de déterminer une catégorie de "désaccord» (dissent) avec le cours officiel qui ne soit ni la protestation plus ou moins violente des masses incontrôlées, immortalisées par Vladimir Kozlov, ni la dissidence politique de l'époque brejnévienne. Il concentre son analyse sur la réception du « discours secret » de HruŠčev au XXe Congrès, déjà bien étudiée ; mais il passe sous silence les réactions scandalisées des tenants de Stalin. L'article ne traitant pas des dissensions exprimées par les nationalistes et les croyants, l'auteur peut conclure: « La majorité des désaccords était essentiellement procommuniste» (p. 176).

Joshua Andy s'attaque à une question difficile à traiter dans l'état actuel des sources : le rôle de l'armée lors de l'écrasement de la manifestation de Novočerkassk (1962). Sans apporter d'éléments factuels nouveaux (on ne sait toujours pas précisément qui a ouvert le feu sur la foule le 2 juin), l'auteur affirme que l'armée régulière a défendu son éthique militaire en refusant de tirer sur des manifestants désarmés et de jouer un rôle policier, 
bien qu'il ne conteste pas que certaines unités de l'armée aient pu participer au massacre aux côtés de la police. Il n'écarte pas non plus une seconde motivation pouvant expliquer la retenue de l'armée et cite l'allégation selon laquelle la troupe était ethniquement russe et ne pouvait donc se résoudre à tirer sur une foule d'ouvriers slaves, alors que les troupes du MVD et du KGB, non slaves, n'auraient pas eu ces scrupules. L'argumentation sur un sujet aussi grave est confuse et peu probante.

11 Proposant une histoire institutionnelle des syndicats, Junbao Jo travaille à partir de la presse pour montrer que les syndicats évoluent entre deux impératifs contradictoires, protection du bien-être ouvrier et mobilisation pour la production. Ce schéma d'analyse trop large, une mauvaise maîtrise du contexte social, économique et politique du dégel, ainsi que l'absence de sources d'archives, interdisent toute approche sérieuse du problème.

12 Précisons que le prix de l'ouvrage - 80 livres sterling - rend inaccessibles au public ces articles qu'il aurait été plus opportun de publier sur un site Internet universitaire.

\section{NOTES}

1. Polly JONES, ed., The Dilemmas of De-Stalinization: Negotiating Cultural and Social Change in the Khrushchev Era, Londres-New York : Routledge, 2006. 